\title{
The legacy of the hadron physics programme at COSY
}

\author{
Colin Wilkin ${ }^{1, \star}$ \\ ${ }^{1}$ UCL, Gower Street, London WC1E 6BT, U.K.
}

\begin{abstract}
A brief review is presented of some elements of the twenty years of hadronic physics research at the COoler SYnchrotron (COSY).
\end{abstract}

\section{Introduction}

At the end of 2014 the priorities of the Institut für Kernphysik Jülich switched from the investigation of hadronic processes to the study of a variety of symmetry-breaking reactions. A review of the activities at the COSY accelerator and storage ring is currently being prepared [1] and this will culminate by highlighting ten specific programmes, viz.

1. Proton-proton elastic scattering

2. The WASA dibaryon

3. Neutron-proton elastic scattering

4. Large acceptance hyperon production

5. The hyperon cusp

6. $\eta$-mesic nuclei

7. Non-strange meson production in nucleon-nucleon collisions

8. Kaon pair production

9. Determination of the mass of the $\eta$ meson

10. Amplitude analysis of $N N \rightarrow\{p p\}_{S} \pi$ at $353 \mathrm{MeV}$

In the time available, I cannot discuss all of them — the rest will be found, along with many many references, in the review article [1].

\section{Proton-proton elastic scattering}

There have been stupendous advances at COSY in the study of $p p$ elastic scattering using the EDDA, ANKE, and KOALA detectors. These all involved measurements with very thin targets inside the COSY ring, where double-scattering experiments were impractical. Hence only initial spin degrees of freedom could be studied. EDDA identified elastic scattering by demanding that the laboratory polar angles of fully coplanar events satisfy

$$
\cot \theta_{\text {lab }}^{1} \cot \theta_{\text {lab }}^{2}=1+T_{p} / 2 m_{p},
$$

^A European Physical Journal A special lecture. e-mail: c.wilkin@ucl.ac.uk 
where $T_{p}$ is the beam laboratory kinetic energy and $m_{p}$ the proton mass.

Measurements of the differential cross section, proton analysing power, and spin-correlations were carried out during acceleration (and deceleration) over a continuum of energies from 230 to $2590 \mathrm{MeV}$. But, since both protons were detected, data were only available for $\theta_{c m} \geq 35^{\circ}$. Excitation functions for these observables at fixed angle are shown in Fig. 1.
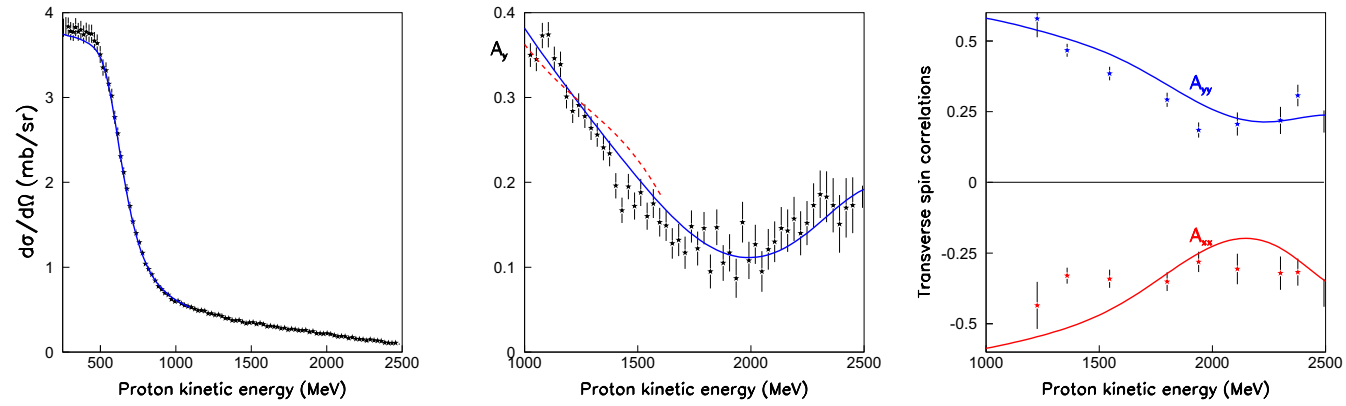

Figure 1. Left: EDDA measurements of the differential cross section for $p p$ scattering at $\theta_{c m}=89^{\circ}$ [2]. Middle: Analysing power in $p p$ elastic scattering measured with EDDA at $\theta_{c m}=56^{\circ}$ [4]. The dashed (solid) curve represents SAID solutions [3] before (after) the EDDA measurements. Right: Transverse spin correlations at $\theta_{c m}=57.5^{\circ}[5]$ compared to the SAID SP07 solution [3].

ANKE investigated $p p$ elastic scattering at smaller angles than EDDA by measuring one final proton and its energy/momentum. The results shown in Fig. 2 were obtained by detecting the fast proton in a magnetic spectrometer or the slow recoil in a pair of silicon tracking telescopes [6, 7]. Even smaller momentum transfers could be investigated using the KOALA detector [8], though the data shown in Fig. 2 are not yet reliably normalised. Nevertheless, it is clear that COSY has completely revolutionised the understanding of $p p$ scattering up to $3 \mathrm{GeV}$.
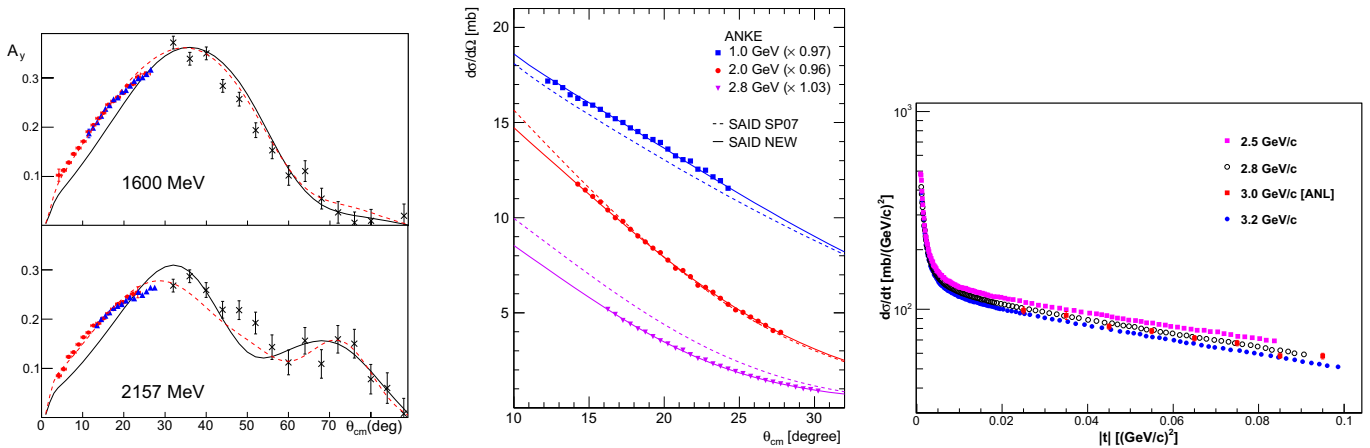

Figure 2. Left: ANKE measurements of the analysing power in $p p$ elastic scattering at two energies (circles/triangles) [6] compared to EDDA (crosses) data [4] and the SAID SP07 fit (solid line) [3] and a revised one (dashed). Middle: ANKE $p p$ differential cross sections [7], scaled by factors within the overall uncertainties, compared to the SAID SP07 solution (dashed) [3] and modified fit (solid line). Right: Preliminary values of the $p p$ differential cross section measured with the KOALA detector [8] at very small momentum transfers $t$. 


\section{The WASA dibaryon}

The search for dibaryons has a long and generally frustrating history. The inspiration came from six-quark bag models that predict several states. But the only confirmed dibaryon was the deuteron, where the relevant degrees of freedom are (probably) nucleons and pions. The WASA collaboration at CELSIUS and COSY measured the total cross section for quasi-free $n p \rightarrow d \pi^{0} \pi^{0}$ by using a deuteron beam or a deuterium target. The c.m. energy $W$ is spread by the Fermi momentum but, by reconstructing the whole event, $W$ could be evaluated with some precision. The very impressive peak of Fig. 3 at $W=2.38 \mathrm{GeV}$ with $\Gamma=70 \mathrm{MeV}$ was obtained in three experiments [9-11]. It was suggested that this was a dibaryon, $d^{*}(2380)$. As shown in the middle panel, the $d^{*}(2380)$ is seen also in $p n \rightarrow d \pi^{+} \pi^{-}$[10]. The right panel shows that the inclusion of the $d^{*}(2380)$ improves considerably the SAID description of the analysing power in $\vec{n} p$ quasi-elastic scattering [12].
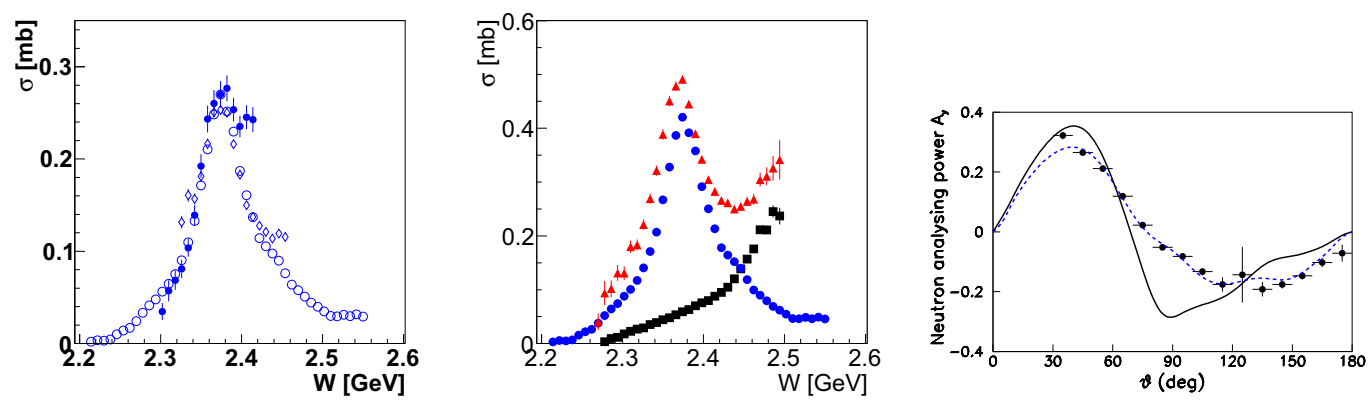

Figure 3. Left: Total cross section for the quasi-free $p n \rightarrow d \pi^{0} \pi^{0}$ reaction as a function of the total energy $W=$ $\sqrt{s}$ [9-11]. Middle: Total cross section for $p n \rightarrow d \pi^{+} \pi^{-}$(red triangles) and its decomposition into isoscalar (blue circles) and isovector parts (black squares) [10]. Right: Angular distribution of the $n p$ quasi-elastic analysing power in the $W=2.377 \mathrm{GeV}$ region [12]. The (black) solid line is the suitably averaged SP07 prediction [3], whereas a SAID solution that includes the dibaryon is shown by the (blue) dashed line.

Though there is still some lingering doubt whether the $d^{*}(2380)$ is really a dibaryon resonance, it is a good working hypothesis that must be tested further. If it exists, is it a 6-quark state or is it a bound state of $\Delta(1232) \Delta(1232)$ ? If $\Delta \Delta$, why is the width so narrow? Even if it turns out not to be a dibaryon, it is still a very important observation in our field and it should be tested in an independent experiment with an independent analysis.

\section{Hyperon production}

By detecting the $K^{+}$and $p$ from $p p \rightarrow K^{+} p X$ one can see peaks from $\Lambda$ and $\Sigma^{0}$ production - but there are significant physics backgrounds due to the misidentification of the direct proton. Thus the proton could have arisen from the decay of a $\Lambda$, a $\Sigma^{0}$, or even a $\Sigma^{+}$. These contributions are less troublesome near threshold where COSY-11 and ANKE have reasonable acceptance. At high excess energy $Q$ (the total c.m. kinetic energy in the final state), the larger acceptance of COSY-TOF is required and this division of effort between low and high $Q$ can be seen in Fig. 4 (left).

The curve for $\Sigma^{0}$ production corresponds to pure three-body phase space whereas that for the $\Lambda$ shows evidence for a strong $\Lambda p$ final state interaction (FSI). This is seen more clearly in the right 
panel, which shows the ratio of $\Lambda$ to $\Sigma^{0}$ production near threshold. The FSI hypothesis suggests that this should vary as $C /(1+\sqrt{1+Q / B})^{2}$ and the data are well fit with $B \approx 5.2 \mathrm{MeV}$.
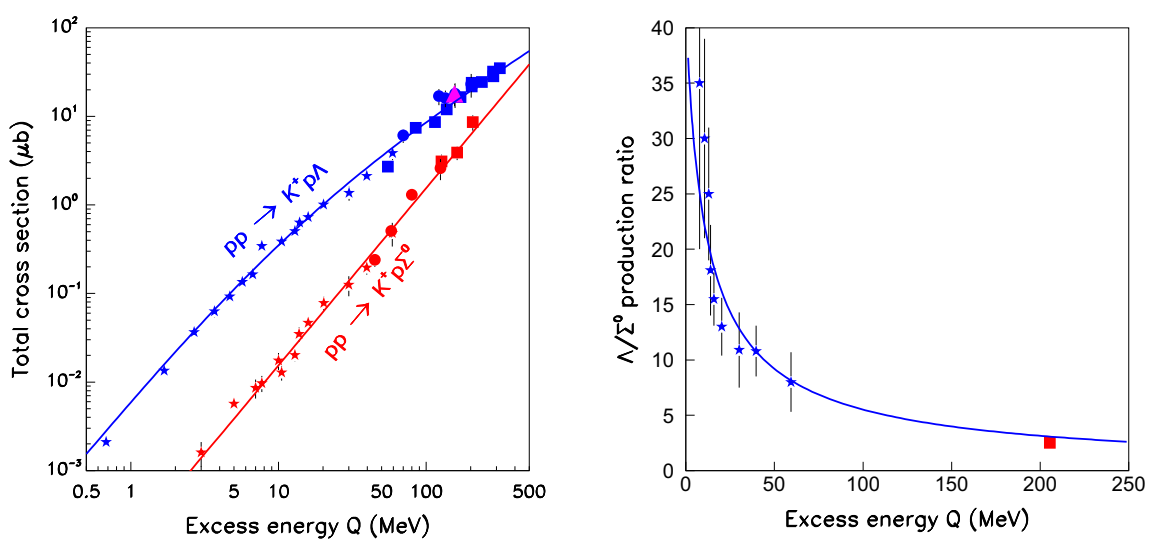

Figure 4. Left: Total cross sections for the $p p \rightarrow K^{+} p \Lambda$ and $p p \rightarrow K^{+} p \Sigma^{0}$. The stars at low $Q$ were obtained at COSY-11 whereas the squares at high $Q$ were taken with the higher acceptance COSY-TOF detector. The curves indicate a significant FSI in the $\Lambda p$ system. This is made even clearer in the ratio of the production cross sections shown in the right panel.

The high geometric acceptance of the COSY-TOF detector allowed the collaboration to investigate a wealth of differential distributions for hyperon production, including polarisation measurements but the one that really fascinates me is the cusp effect shown in Fig. 5.

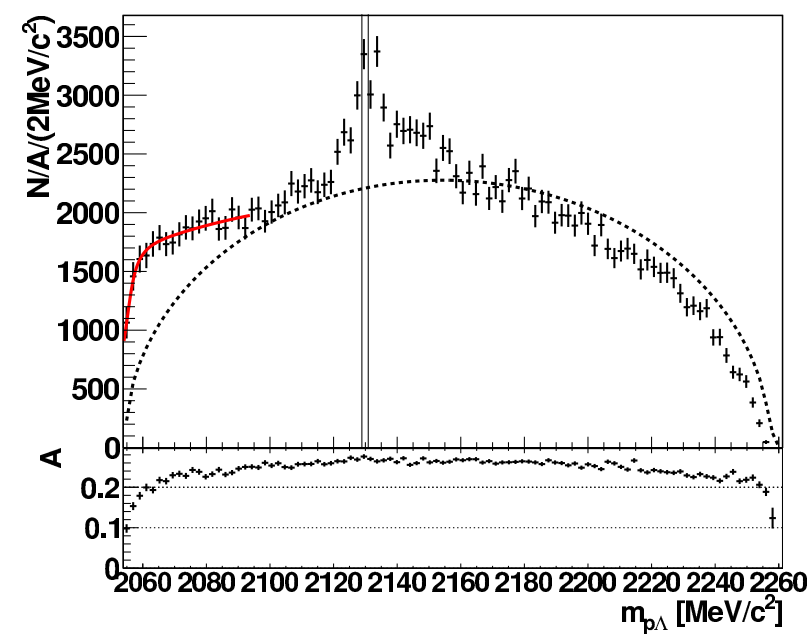

Figure 5. The distribution in $p \Lambda$ invariant mass from the $p p \rightarrow K^{+} p \Lambda$ reaction at $2.95 \mathrm{GeV} / c$ [13]. The vertical lines indicate the positions of the two $\Sigma N$ thresholds. The solid (red) line at low invariant masses represents the data used in the COSY-TOF scattering length determination. 
In unambiguous $p p \rightarrow K^{+} p \Lambda$ events the COSY-TOF collaboration found a very pronounced peak in the vicinity of $m_{p \Lambda} \approx m_{p}+m_{\Sigma^{0}}$ or $m_{n}+m_{\Sigma^{+}}$. Since the acceptance $A$ is smooth in this region, this must be a physics effect, linked to the coupling between the $\Lambda p$ and $\Sigma N$ channels. One is therefore seeing the effects of $p p \rightarrow K^{+} N \Sigma$ followed by $N \Sigma \rightarrow p \Lambda$. This contribution interferes with direct $p p \rightarrow K^{+} p \Lambda$ production and, since it is an interference effect, there is no reason for it to look like a simple resonance peak.

Such a cusp is very common in reactions involving strange particles but they are very hard to model. If it is only the $K^{+}$that is detected it is not possible to distinguish true $\Sigma$ production from $\Lambda$ production where there is a cusp at the $\Sigma N$ thresholds. For example, the HIRES collaboration measured $p p \rightarrow K^{+} X$ at $\theta_{K}=0^{\circ}$ and found a big jump at $M_{X} \approx M_{\Sigma}+M_{N}$ [14]. How much of this was due to $\Sigma$ production - impossible to tell in an inclusive measurement.

\section{$5 \eta$-mesic nuclei}

It was suggested many years ago [15] that the strong attraction between the isoscalar $\eta$ meson and nucleons might be sufficient to let the $\eta$ bind to some nuclei. These would be at best quasi-bound states because they could decay through pion or nucleon emission. The clearest signal for such a possible state is to be found in measurements of the $d p \rightarrow{ }^{3} \mathrm{He} \eta$ cross section carried out near threshold at COSY-11 [16] and ANKE [17], where the data suggest that there is a pole in the $\eta^{3} \mathrm{He}$ elastic scattering amplitude in the complex $Q$ plane for $|Q|<0.5 \mathrm{MeV}$.
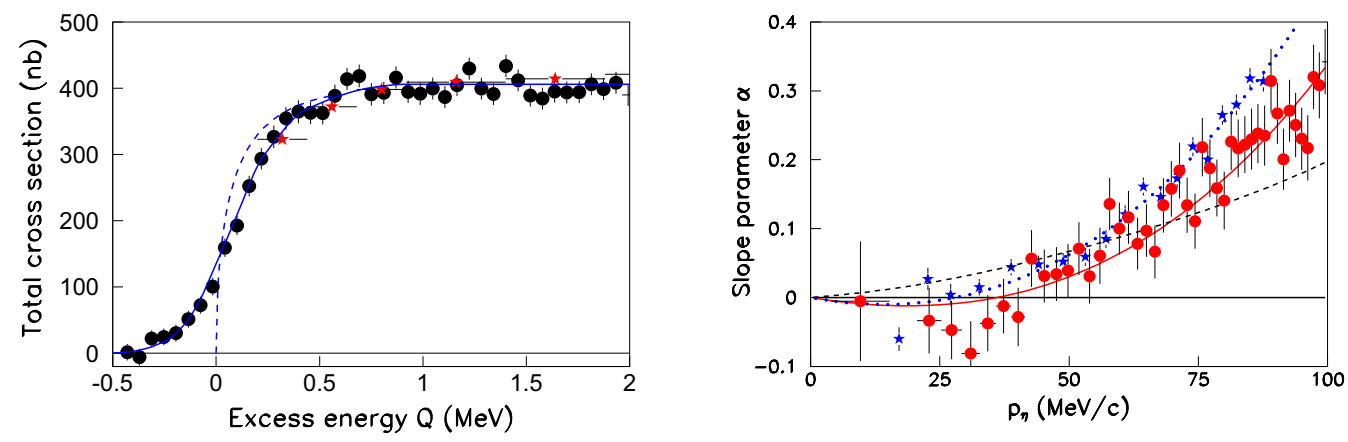

Figure 6. Left: Near-threshold $d p \rightarrow{ }^{3} \mathrm{He} \eta$ total cross sections measured at ANKE (black circles) [17] and COSY-11 (red stars) [16]. The solid curve is the FSI fit to the ANKE data with a $171 \mathrm{keV}$ smearing in $Q$. The dashed curve shows what the data would look like if all sources of smearing could be eliminated. Right: Slope parameter as a function of the $\eta$ c.m. momentum. The ANKE (red circles) and COSY-11 (blue stars) data are compared to fits (solid red curve and blue dots) where the phase variation of the $s$-wave amplitude is taken into account [18]. If the phase variation is neglected, the best fit (black dashed curve) fails to describe the data.

As can be seen from Fig. 6, the cross section jumps to its plateau value for $Q \lesssim 0.5 \mathrm{MeV}$ and the jump is even more abrupt if the effects of the smearing of the beam momentum are taken into account. These data suggest that there is a pole at an $\eta$ c.m. momentum of $p_{1} \approx(-5 \pm i 19) \mathrm{MeV} / c$. The \pm sign here reflects the fact that above-threshold data can never distinguish between a "bound" and a "virtual" state. 
The rapid change of phase due to the pole in the $s$-wave amplitude affects the $s-p$ interference and hence the relative slope of the differential cross section at $90^{\circ}$. It is shown in the right panel of Fig. 6 that the momentum dependence of the slope is better described if the pole is included [18]. This is by far the best signal in the literature for nearby $\eta$-nucleus poles but it doesn't tell us whether it lies on the bound or virtual sheet.

The same reaction was used as the basis of a measurement of the mass of the $\eta$ meson [19]. For this purpose the momentum of the circulating deuteron beam in COSY was measured with a relative precision of about $3 \times 10^{-5}$ by inducing an artificial depolarising resonance, whose position could be evaluated very accurately. The value obtained, $m_{\eta}=\left(547.873 \pm 0.005_{\text {stat }} \pm 0.023_{\text {syst }}\right) \mathrm{MeV} / c^{2}$, is marginally better than any of the other modern measurements.

\section{Kaon pair production}

The measurements of $p p \rightarrow p p K^{+} K^{-}$by the COSY-11 collaboration [20] suggested that the $K^{-}$is preferentially attracted to one (or both) of the final protons. This was repeated with higher statistics at ANKE [21], where the initial drive came from the study of $\phi$ production in $p p \rightarrow p p\left(\phi \rightarrow K^{+} K^{-}\right)$. To see the effect more clearly, define the ratios of cross sections with respect to the $K^{ \pm} p$ and $K^{ \pm} p p$ invariant masses:

$$
R_{K p}=\frac{\mathrm{d} \sigma / \mathrm{d} M_{K^{-}} p}{\mathrm{~d} \sigma / \mathrm{d} M_{K^{+}}}, \quad R_{K p p}=\frac{\mathrm{d} \sigma / \mathrm{d} M_{K^{-}} p p}{\mathrm{~d} \sigma / \mathrm{d} M_{K^{+}} p p},
$$

whose experimental values at $Q=24 \mathrm{MeV}$ are shown in Fig. 7 [21].
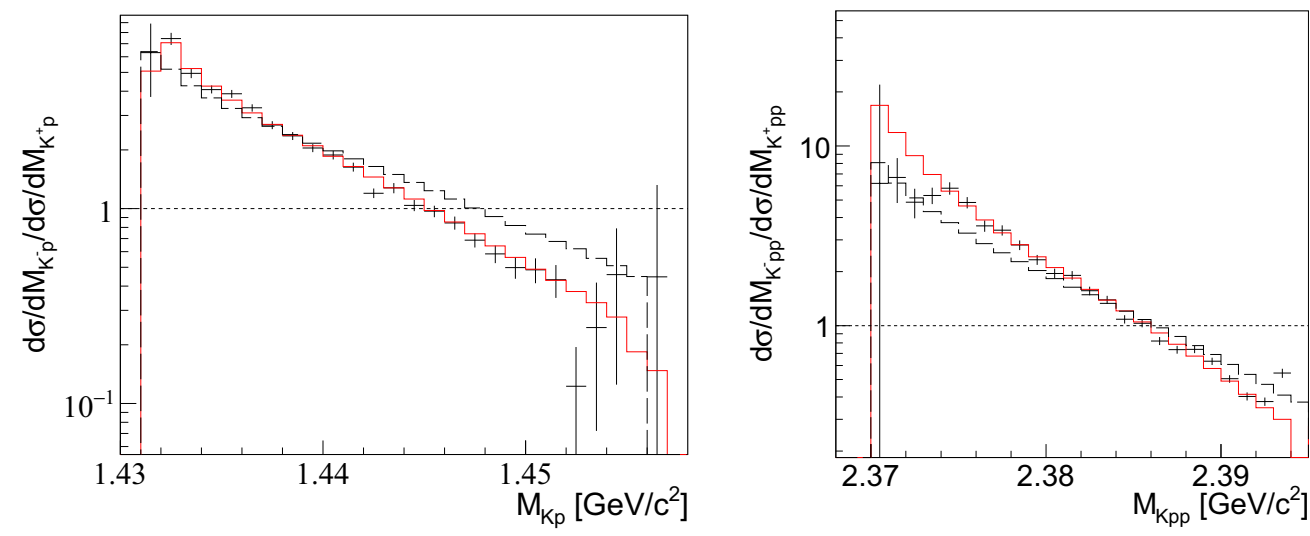

Figure 7. The ratios $R_{K p}$ and $R_{K p p}$ of Eq. (2) at $Q=24 \mathrm{MeV}$ [21]. The red solid and broken black histograms represent estimations that take into account $K^{-} p, p p$ and $K^{+} K^{-}$final state interactions with $a_{K^{-}} p=2.45 i \mathrm{fm}$ and $a_{K^{-} p}=1.5 i \mathrm{fm}$, respectively.

There can be no rigorous evaluation of an enhancement factor when three or more particles interact in the final state. The estimates made in Fig. 7 assume that the overall enhancement is the product of simple FSI factors in the different kinematic variables. With a reasonable value of the effective $K^{-} p$ scattering length, such a simple model does explain the enormous variations of the ratios as functions of the $K p$ and $K p p$ masses. Any relationship between this and a possible bound $K^{-} p p$ system is a 
matter for speculation. There is, however, almost certainly a connection to the $p p \rightarrow K^{+} p \Lambda(1405)$ reaction, which has also been studied at COSY [22].

\section{Conclusions}

The results presented here represent only a very small part of the successes in hadronic physics achieved at COSY over the last twenty years. Their compression into a few pages will certainly lead to distortions in the text and the references. It is to be hoped that the full review article of Ref. [1] will have fewer flaws.

Support from both the conference organisers and the European Physical Journal are gratefully acknowledged.

\section{References}

[1] C. Wilkin, Invited review for Eur. Phys. J. in preparation

[2] D. Albers et al., Eur. Phys. J. A 22, 125 (2004)

[3] R.A. Arndt, W.J. Briscoe, I.I. Strakovsky, R.L. Workman, Phys. Rev. C 76, 025209 (2007)

[4] M. Altmeier et al., Eur. Phys. J. A 23, 351 (2005)

[5] F. Bauer et al., Phys. Rev. C 71, 054002 (2005)

[6] Z. Bagdasarian et al., Phys. Lett. B 739, 152 (2014)

[7] D. Mchedlishvili et al., Phys. Lett. 755, 92 (2016)

[8] Q. Hu et al., Eur. Phys. J. A 50, 156 (2014)

[9] P. Adlarson et al., Phys. Rev. Lett. 106, 242302 (2011)

[10] P. Adlarson et al., Phys. Lett. B 721, 229 (2013)

[11] P. Adlarson et al., Eur. Phys. J. A 52, 147 (2016)

[12] P. Adlarson et al., Phys. Rev. Lett. 112, 202301 (2014)

[13] M. Röder et al., Eur. Phys. J. A 49, 157 (2013)

[14] A. Budzanowski et al., Phys. Lett. B 692, 10 (2010)

[15] Q. Haider and L.C. Liu, Phys. Lett. B 172, 257 (1986)

[16] J. Smyrski et al., Phys. Lett. B 649, 258 (2007)

[17] T. Mersmann et al., Phys. Rev. Lett. 98, 242301 (2007)

[18] C. Wilkin et al., Phys. Lett. B 654, 92 (2007)

[19] P. Goslawski et al., Phys. Rev. D 85, 112011 (2012)

[20] P. Winter et al., Phys. Lett. B 635, 23 (2006)

[21] Q.J. Ye et al., Phys. Rev. C 87, 065203 (2013)

[22] I. Zychor et al., Phys. Lett. B 660, 167 (2008) 